\title{
Impact of immunotherapy use in patients with stage IV pancreatic carcinoma
}

\author{
Achuta K. Guddati ${ }^{1}$, Takefumi Komiya ${ }^{2}$, Sunny J. Patel ${ }^{1}$, Neil Sharma ${ }^{3}$, Emily Powell ${ }^{4}$ \\ ${ }^{1}$ Division of Hematology/Oncology, Augusta University, Augusta, GA, USA; ${ }^{2}$ Hematology/Medical Oncology, Parkview Cancer Institute, Fort \\ Wayne, IN, USA; ${ }^{3}$ Interventional GI Oncology, Fort Wayne, IN, USA; ${ }^{4}$ Parkview Research Center, Mirro Center for Research and Innovation, Fort \\ Wayne, IN, USA \\ Contributions: (I) Conception and design: AK Guddati, T Komiya, E Powell; (II) Administrative support: T Komiya, N Sharma, E Powell; (III) \\ Provision of study materials or patients: AK Guddati, T Komiya, E Powell; (IV) Collection and assembly of data: T Komiya, E Powell; (V) Data \\ analysis and interpretation: AK Guddati, T Komiya, SJ Patel, N Sharma, E Powell; (VI) Manuscript writing: All authors; (VII) Final approval of \\ manuscript: All authors. \\ Correspondence to: Dr. Takefumi Komiya, MD, PhD. Division of Hematology/Oncology, Parkview Cancer Institute, 11050 Parkview Circle, Fort \\ Wayne, IN 46845, USA. Email: takefumi.komiya@parkview.com.
}

Background: Most patients with pancreatic cancer have non-resectable disease at the time of diagnosis and usually die within 6-12 months. Despite indications in other solid tumors, the role of immunotherapy (IO) is unknown for late stage, advanced pancreatic cancer.

Methods: Using the National Cancer Database (NCDB), cases of Stage IV pancreatic cancers diagnosed in the period of 2014-2016 with at least 30-day follow up were retrospectively analyzed. The following clinical demographics were included: age (younger than $70 v s$. older than 70), sex (male vs. female), race (whites $v s$. others), insurance (uninsured vs. insured), type of institution (academic vs. nonacademic), liver metastasis (yes $v s$. no), lung metastasis (yes $v s$. no), external beam radiation (yes $v s$. no), systemic chemotherapy (yes $v s$. no) and IO (yes vs. no). survival analysis was performed using Kaplan-Meier curves and Log-rank tests. Multivariable Cox proportional hazard models and propensity score matching analysis were also utilized. A P value $<0.05$ was considered significant.

Results: Among 25,596 eligible cases, 163 patients were treated with IO. A significant association between the use of IO and several clinical demographics (age <70, academic institution, adenocarcinoma, lung metastasis, radiation, chemotherapy) was noted. Chemotherapy was administered in 133 (82\%) and 16,342 $(64 \%)$ of cases in the IO and non-IO groups, respectively. Use of IO was associated with improved overall survival (OS) in both univariate and multivariate analyses $(\mathrm{P}<0.0001$ for each). Median OS (in months) was 12.2 in the IO group vs. 5.8 in the non-IO group. Landmark analysis in the IO group showed 12 and 24-month survival of $51.0 \%$ and $20.0 \%$ respectively, as compared with $28.2 \%$ and $11.9 \%$ in the non-IO group. Propensity score matching analysis also demonstrated a trend toward improved OS in IO group $(\mathrm{P}=0.0753)$. Median survival was 12.2 and 8.9 months, respectively.

Conclusions: This retrospective data analysis using a large cancer database suggests that use of IO could improve survival in patients with advanced pancreatic cancer. More studies will be needed in the future to validate these results.

Keywords: Immunotherapy (IO); propensity score matching; pancreatic cancer; PD-1

Submitted Apr 21, 2020. Accepted for publication Jul 31, 2020.

doi: 10.21037/jgo-20-191

View this article at: http://dx.doi.org/10.21037/jgo-20-191 


\section{Introduction}

Pancreatic carcinoma is one of the deadliest malignancies, ranking third in cancer-related deaths in the United States with projections to become the second leading cause by 2030 (1). The incidence of pancreatic cancer has been steadily increasing over the years and in 2020 itself, nearly 57,600 incident cases are projected. In fact, similar numbers have unfortunately been predicted for pancreatic cancerrelated mortality with approximately 47,050 estimated deaths as well (2). The prognosis of pancreatic cancer remains dismal. The overall 5 -year survival rate is $9 \%$ with an expected survival of less than one year $(2,3)$. The low survival rate is attributed to many factors, of which the late presentation of many patients is likely the most important. Due to the relative asymptomatic nature of the initial stages of the disease, the majority of patients with pancreatic cancer have metastatic disease upon diagnosis or disease that has aggressively spread to common sites, such as the liver or peritoneal cavity $(3,4)$. Less than $20 \%$ of patients present with localized, resectable tumors, and while surgical resection is regarded as the only treatment for cure, most cancers recur. Treatment options for advanced metastatic disease are scarce. Chemotherapy remains a mainstay treatment with palliative supportive care recognized as an important adjunct (5).

Although no adequate therapy has been found for advanced pancreatic cancer, immunotherapy (IO) may hold promise. Optimism surrounds IO as a viable therapeutic approach for the management of solid tumors. Recently, phase III clinical trials have demonstrated the efficacy of IO in the management of solid cancers (6). As a result, the Food and Drug Administration has approved pembrolizumab and nivolumab for head and neck cancer, renal cancer, melanoma, and lung cancer $(7,8)$. In pancreatic cancer specifically, multiple small-sized studies and clinical trials involving immune-based strategies are ongoing with preliminary positive results $(9,10)$. Although no conclusive data has been established, a formal analysis has not been conducted and the role of this form of therapy still remains unknown without a convincing consensus. Because of this cancer's therapeutic resistance and the lack of definitive studies, the utilization of IO in this patient population has not been well-assessed. In addition, analyses regarding the demographic characteristics and clinical factors associated with and comparing patients who may or may not have received IO have not been performed to date. The current study was undertaken to perform such analysis as well as investigate the impact of IO on the overall survival (OS) of patients with advanced stage IV pancreatic cancer. We present the following article in accordance with the STROBE checklist. Available at: http://dx.doi. org/10.21037/jgo-20-191.

\section{Methods}

\section{Database and study population}

This was a retrospective analysis of hospital-based data obtained from the National Cancer Database (NCDB). The NCDB is a joint project established in 1989 of the Commission on Cancer $(\mathrm{CoC})$ of the American College of Surgeons and the American Cancer Society. Since its inception, the NCDB has become one of the largest cancer registries that comprises approximately $70 \%$ of cancer cases in the United States from over 1,500 CoC-accredited centers. It is one of the most commonly used sources to track trends in cancer care. Data is collected on various information, including patient factors, Charlson-Deyo comorbidity scores, tumor characteristics, staging details, surgical and adjuvant treatments, and clinical outcomes $(11,12)$. The CoC's NCDB and the hospitals participating in this database are the source of the de-identified data used herein; they have not verified and are not responsible for the statistical validity of the data analysis or the conclusions derived by the investigators. This study was exempt from review by the Institutional Review Board and patient consent was waived due to the utilization of de-identified data. This study is in accordance with the Declaration of Helsinki (as was revised in 2013).

Patients diagnosed with stage IV pancreatic cancer with at least a 30-day follow-up from January 1, 2014 to December 31, 2016 at CoC-accredited centers were identified and included in the study. A vast majority [23,795 of $25,596(93 \%)$ in all groups, 152 of $163(93 \%)$ in IO group] had M1. Tumor metastatic stage was defined based upon the American Joint Committee on Cancer 7th edition staging manual. Patients were identified using the appropriate International Classification of Diseases for Oncology, $3^{\text {rd }}$ edition (ICD-O-3) codes based on histology and anatomic primary site. Demographic information consisting of age, gender, race, insurance coverage, and comorbidity along with hospital institution characteristics were extracted from patients that met this criterion. Comorbidity was evaluated using Charlson-Deyo scores. Tumor characteristics involving the histology as well as 
primary and metastatic sites of lesions and treatments utilized for therapeutic management (chemotherapy, external beam radiation, IO) were recorded as well. The NCDB does not identify specific therapy agents used for treatment; instead the general utilization and receipt of respective treatment modalities was logged. Information in treatment was available via NCDB only for first-course of therapy. Treatments after progression on the initial therapy were not collected by NCDB.

\section{Statistical analysis}

Descriptive statistics of demographic, clinical, and treatment characteristics were recorded as categorical variables. Pearson $\chi^{2}$ tests were utilized to make comparisons. OS was measured from the date of diagnosis to the time of last follow-up or death. For better correlation with OS, the study was restricted to patients with at least a 30-day followup. Univariate survival analysis was performed using Kaplan Meier curves. Statistical comparisons of survival between groups treated with and without IO were performed with log-rank tests. Cox proportional hazards regression modeling was used for multivariate survival analysis according to IO treatment utilization adjusted for age, sex, race, institution, insurance coverage, Charlson-Deyo score, cancer histology, tumor primary and metastatic sites, and treatment modalities. Because of the size difference between the IO-positive and IO-negative cohorts, we also utilized a propensity score analysis to account for and potentially reduce the effects of potential biases and confounders in our study (13). All statistical analyses were performed using JMP software version 13 (SAS Institute, Inc., Cary, NC, USA). Adjusted hazard ratios (HR) and $95 \%$ confidence interval (CI) are reported, with $\mathrm{P}$ value $<0.05$ used to indicate statistical significance.

\section{Results}

\section{Population characteristics}

A total of 25,596 cases met our inclusion criteria. Of these cases, $163(0.6 \%)$ patients were treated with IO. A significant association between the use of IO and several clinical demographics was noted. Patients treated with IO tended to be $<70$ years old, receive care at an academic institution, have adenocarcinoma with lung metastasis, and receive radiation and chemotherapy for treatments. Chemotherapy was administered in 133 (82\%) and 16,342 (64\%) of cases in IO and non-IO groups, respectively. Tables 1 and 2 illustrate the summary statistics for demographic, hospital, and clinical factors including multiagent chemotherapy without and with propensity score matching.

\section{Survival impact of IO}

Use of IO was associated with improved OS in both univariate and multivariate analyses $(\mathrm{P}<0.0001$ for each $)$ (Table 3). Among the entire cohort, median OS (in months) as measured by Kaplan-Meier was 12.2 in the IO group vs. 5.8 in the non-IO group. Landmark analysis in the IO group showed 12 - and 24-month survival of $51.0 \%$ and $20.0 \%$ respectively, as compared with $28.2 \%$ and $11.9 \%$ in the non-IO group (Figure 1). After adjusting for imbalanced covariates among IO-treated and non-IO-treated cohorts, propensity score matching analysis assessing receipt of IO continued to demonstrate a trend toward improved OS in the IO group $(\mathrm{P}=0.0753)$. Median survival was 12.2 and 8.9 months, respectively (Figure 2).

\section{Discussion}

Pancreatic carcinoma is an extremely lethal malignancy that is especially difficult to treat. Only a small percentage of the patient population can undergo surgical resection. The majority of patients are diagnosed with advanced stage IV unresectable disease and life expectancy is in months. With traditional chemotherapeutic agents providing limited survival advantage and in the absence of targeted agents, IO has gained interest but the potential benefit of it remains unknown. This nationwide analysis examined survival outcomes for patients with unresected advanced pancreatic cancer who received IO versus those who did not. The results of this study suggest an OS benefit for IO (median survival, 12.2 vs. 5.8 months).

There is a paucity of large clinical studies that specifically address the efficacy of IO in advanced pancreatic cancer. Despite lacking significant power and size, a few studies have shown promising results. Beatty et al. investigated co-stimulatory immunomodulatory CD 40 agonists in 21 patients with locally advanced or metastatic pancreatic cancer. They demonstrated that this therapy with the chemotherapeutic agent, gemcitabine, improved median OS compared to gemcitabine alone (7.4 vs. 5.7 months). One patient with metastatic hepatic lesions was even able to undergo surgical resection after therapy reduced the tumor size by $64 \%$ and eradicated all hepatic metastases (14). In 
Table 1 Clinical characteristics of cases selected for analysis

\begin{tabular}{|c|c|c|c|c|}
\hline Variable & \multicolumn{2}{|c|}{ Immunotherapy } & Total & $P$ value \\
\hline Total & $163(100 \%)$ & $25,433(100 \%)$ & $25,596(100 \%)$ & \\
\hline $70+$ & $44(27 \%)$ & $10,738(42 \%)$ & $10,782(42 \%)$ & \\
\hline$<70$ & 119 (73\%) & 14,695 (58\%) & $14,814(58 \%)$ & \\
\hline Male & $86(53 \%)$ & $13,402(53 \%)$ & $13,488(53 \%)$ & \\
\hline Female & $77(47 \%)$ & $12,031(47 \%)$ & $12,108(47 \%)$ & \\
\hline Race & & & & 0.0156 \\
\hline White & 145 (89\%) & $20,754(82 \%)$ & 20,899 (82\%) & \\
\hline Other & $76(47 \%)$ & $15,458(61 \%)$ & $15,534(61 \%)$ & \\
\hline Insurance & & & & 0.3018 \\
\hline Yes & $161(99 \%)$ & 24,800 (98\%) & 24,961 (98\%) & \\
\hline No & $2(1 \%)$ & $633(2 \%)$ & $635(2 \%)$ & \\
\hline Charlson-Deyo score & & & & 0.3793 \\
\hline $0-1$ & $152(93 \%)$ & 23,222 (91\%) & $23,374(91 \%)$ & \\
\hline $2-3$ & $11(7 \%)$ & $2,211(9 \%)$ & $2,222(9 \%)$ & \\
\hline Histology & & & & 0.0151 \\
\hline Yes & 48 (29\%) & $4,814(19 \%)$ & 4,862 (19\%) & \\
\hline No & $115(71 \%)$ & $20,619(81 \%)$ & $20,734(81 \%)$ & \\
\hline Radiation & & & & $<0.0001$ \\
\hline Yes & $19(12 \%)$ & $1,261(5 \%)$ & $1,280(5 \%)$ & \\
\hline No & $144(88 \%)$ & $24,172(95 \%)$ & $24,316(95 \%)$ & \\
\hline Chemotherapy & & & & $<0.0001$ \\
\hline Yes & $133(82 \%)$ & $16,342(64 \%)$ & $16,475(64 \%)$ & \\
\hline No & $30(18 \%)$ & $9,091(36 \%)$ & $9,121(6 \%)$ & \\
\hline Primary location & & & & 0.0005 \\
\hline Pancreatic Head & $42(26 \%)$ & $9,223(36 \%)$ & $9,265(36 \%)$ & \\
\hline Other & $121(74 \%)$ & $16,210(64 \%)$ & $16,331(64 \%)$ & \\
\hline Multi-agent chemotherapy & & & & $<0.0001$ \\
\hline Yes & $115(71 \%)$ & $12,881(51 \%)$ & $12,996(51 \%)$ & \\
\hline No & $48(29 \%)$ & $12,552(49 \%)$ & $12,600(49 \%)$ & \\
\hline
\end{tabular}

(C) Journal of Gastrointestinal Oncology. All rights reserved. 
Table 2 Univariate and multivariate analyses for OS

\begin{tabular}{|c|c|c|c|c|}
\hline Variable & \multicolumn{2}{|c|}{ Univariate } & \multicolumn{2}{|c|}{ Multivariate } \\
\hline \multicolumn{5}{|l|}{ Age } \\
\hline 20-69 vs. $70+$ & $0.68(0.67-0.70)$ & $<0.0001$ & $0.76(0.74-0.78)$ & $<0.0001$ \\
\hline \multicolumn{5}{|l|}{ Sex } \\
\hline \multicolumn{5}{|l|}{ Race } \\
\hline Others vs. Whites & $0.97(0.94-1.01)$ & 0.1548 & $0.96(0.92-0.99)$ & 0.0200 \\
\hline \multicolumn{5}{|l|}{ Institution } \\
\hline Academic vs. nonacademic & $0.81(0.79-0.83)$ & $<0.0001$ & $0.83(0.80-0.85)$ & $<0.0001$ \\
\hline \multicolumn{5}{|l|}{ Charlson-Deyo score } \\
\hline $0-1$ vs. $2-3$ & $0.72(0.68-0.75)$ & $<0.0001$ & $0.80(0.76-0.84)$ & $<0.0001$ \\
\hline \multicolumn{5}{|l|}{ Histology } \\
\hline Others vs. adenocarcinoma & $0.73(0.71-0.76)$ & $<0.0001$ & $0.64(0.62-0.66)$ & $<0.0001$ \\
\hline \multicolumn{5}{|l|}{ Liver metastasis } \\
\hline No vs. yes & $0.89(0.86-0.92)$ & $<0.0001$ & $0.83(0.80-0.86)$ & $<0.0001$ \\
\hline \multicolumn{5}{|l|}{ Lung metastasis } \\
\hline No vs. yes & $0.85(0.82-0.88)$ & $<0.0001$ & $0.83(0.81-0.86)$ & $<0.0001$ \\
\hline \multicolumn{5}{|l|}{ Radiation } \\
\hline \multicolumn{5}{|l|}{ Primary location } \\
\hline Pancreatic head vs. other & $0.97(0.94-1.00)$ & 0.0396 & $0.97(0.94-1.00)$ & 0.00394 \\
\hline \multicolumn{5}{|l|}{ Immunotherapy } \\
\hline Yes vs. no/unknown & $0.60(0.49-0.71)$ & $<0.0001$ & $0.69(0.57-0.82)$ & $<0.0001$ \\
\hline
\end{tabular}

$\mathrm{HR}$, hazard ratio; $\mathrm{Cl}$, confidence interval; OS, overall survival.

a phase I trial, 49 patients with advanced pancreatic cancer received dendritic cell-based IO with gemcitabine with or without lymphokine activated killer (LAK) IO. Patients who received the combination treatment with LAK therapy were noted to have prolonged survival (15). Both of these studies utilized a combination of IO and chemotherapy to treat cancer. It has been well-recognized that the utilization of chemotherapy to prime the immune system in preparation for IO can be advantageous against cancer.
The above studies confirm that the belief found in other studies that chemo-IO can have synergistic effects that improve OS outcomes (16-18). Our study also indicated that most patients who receive IO also were treated with chemotherapy (likely gemcitabine and protein-bound paclitaxel), confirming that these therapies are being given in combination due to their ability to enhance anti-tumor immune responses in cancer.

Other clinical studies have also looked specifically at 
Table 3 Propensity score matched cases

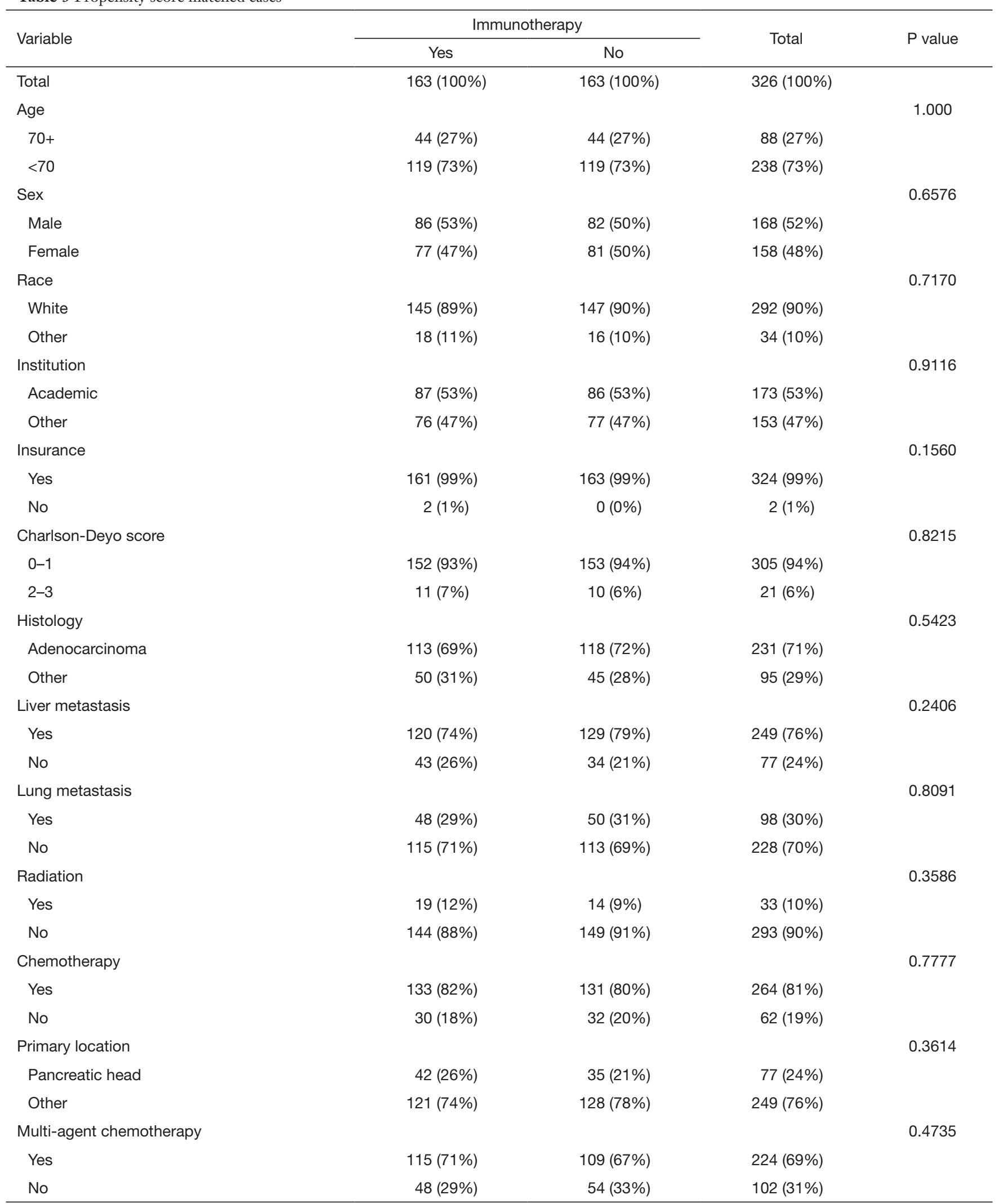




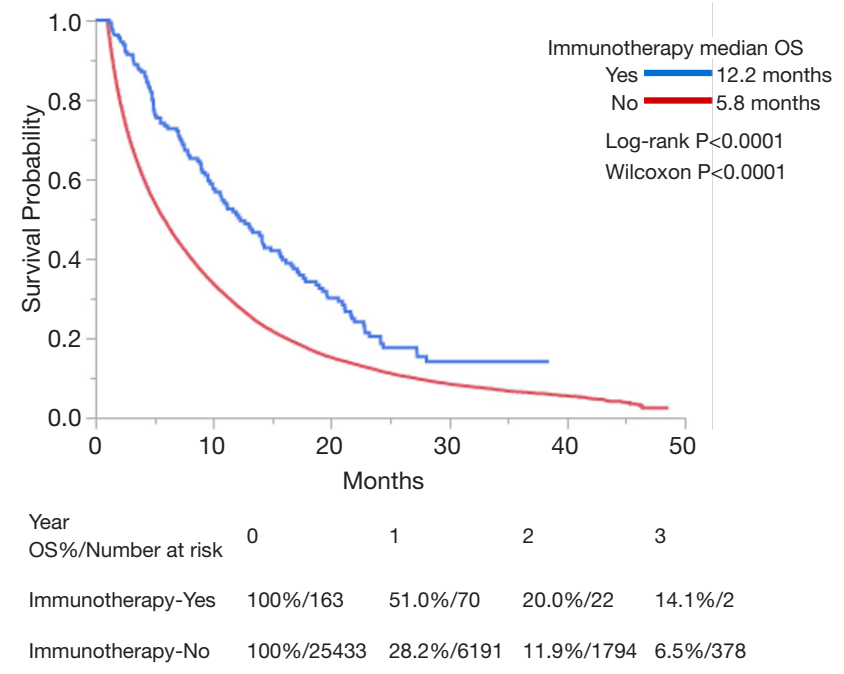

Figure 1 Kaplan-Meier curves of OS for patients with stage IV pancreatic cancer treated with or without IO. IO, immunotherapy; OS, overall survival.
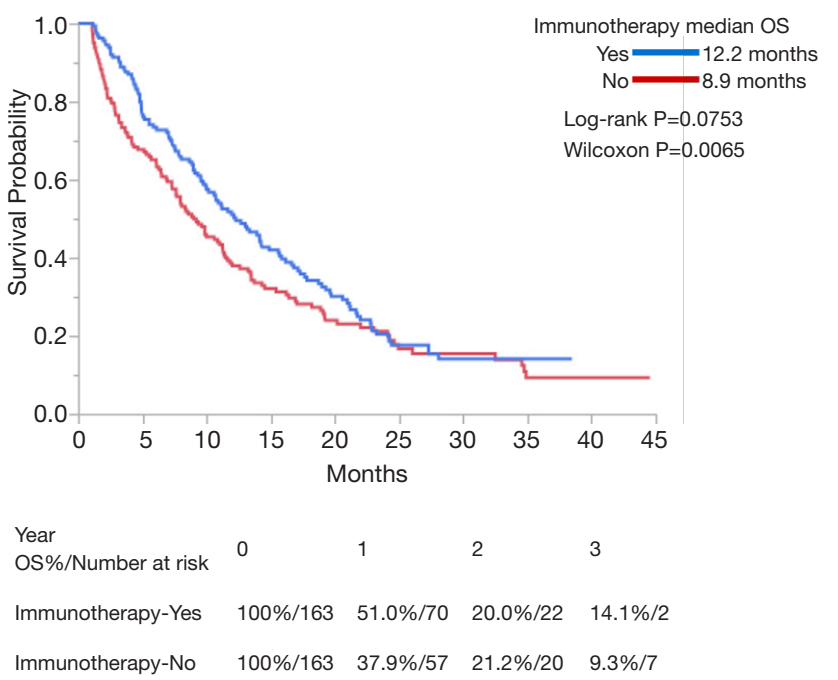

Figure 2 Kaplan-Meier curves of OS for patients with stage IV pancreatic cancer treated with or without IO after propensity score matching. IO, immunotherapy; OS, overall survival.

the use of IO in stage IV pancreatic cancer patients and reported OS benefits similar to our study. One study in which 15 of 20 patients had stage IV pancreatic cancer assessed the efficacy of adoptive IO utilizing dendritic cells and cytotoxic $\mathrm{T}$ lymphocytes. The combinative IO resulted in greater than 20\% 1-year survival for these patients. Five patients were found to have stable disease and one of these patients with liver metastasis maintained stable disease for 6 months. Most impressively, one patient who had multiple lung metastases experienced a complete response that lasted $>5$ years (19). Similarly, Lin et al. found comparable results in patients with unresectable stage IV pancreatic cancer. They noted a significant survival benefit with a higher median OS when cancer ablation treatment was combined with natural killer cell therapy (20). Our study which includes the largest investigation and analysis of stage IV pancreatic cancer patients to date corroborates and validates these studies in demonstrating an OS benefit in patients who received IO.

\section{Strengths and limitations}

This study was powered by a large sample size representative of most cancer cases across the United States. It provided sufficient data to quantify trends and the impact of IO in patients with stage IV pancreatic cancer-an investigation that has been difficult to assess from previous studies and trials. Despite these strengths, the study still has limitations inherent to its retrospective nature and use of a database like the NCDB. One major limitation involves selection bias. Our study found that the patients in the cohort that received IO were younger, relatively healthier with less comorbidity, and likely treated through clinical trials at academic facilities than those who did not receive IO. This is very similar to another study that investigated the role of adjuvant chemo-IO in pancreatic cancer (18). While we attempted to minimize selection bias through propensity score matching analysis, we understand that it cannot be completely nullified. We also realize this along with the small size of the IO-treated patient cohort in comparison to the opposing group may have affected this study's external validity. Limitations associated with the NCDB include possible erroneous coding entries in the dataset and the inability to assess data relating to other endpoints of interest such as treatment toxicity, quality of life, and progression-free survival. Moreover, the NCDB generalized treatment information and did not provide the specific treatment agents used within cases. This limited our ability to further compare outcomes among various known IO agents.

\section{Conclusions}

To our knowledge, this study is the largest investigation to assess the trends and outcomes of patients with advanced, 
stage IV pancreatic cancer who may or may not have received IO. Using the NCDB, this analysis demonstrated a survival benefit with improved OS in patients who received IO. Given the lack of available definitive treatment for advanced pancreatic cancer, our study suggests that IO treatment should be considered and ultimately implemented in this patient population. Our findings warrant the need for future prospective, randomized clinical trials that evaluate outcomes related to long-term survival, quality of life, and overall efficacy of IO for stage IV pancreatic cancer.

\section{Acknowledgments}

We would like to thank Dr. Mariajose Rojas MD for critiquing manuscript.

Funding: None.

\section{Footnote}

Reporting Checklist: The authors have completed the STROBE reporting checklist. Available at: http://dx.doi. org/10.21037/jgo-20-191

Data Sharing Statement: Available at http://dx.doi. org/10.21037/jgo-20-191

Conflicts of Interest: All authors have completed the ICMJE uniform disclosure form (available at: http://dx.doi. org/10.21037/jgo-20-191). The authors have no conflicts of interest to declare.

Ethical Statement: The authors are accountable for all aspects of the work in ensuring that questions related to the accuracy or integrity of any part of the work are appropriately investigated and resolved. This study was exempt from review by the Institutional Review Board and patient consent was waived due to the utilization of de-identified data. This study is in accordance with the Declaration of Helsinki (as was revised in 2013).

Open Access Statement: This is an Open Access article distributed in accordance with the Creative Commons Attribution-NonCommercial-NoDerivs 4.0 International License (CC BY-NC-ND 4.0), which permits the noncommercial replication and distribution of the article with the strict proviso that no changes or edits are made and the original work is properly cited (including links to both the formal publication through the relevant DOI and the license).
See: https://creativecommons.org/licenses/by-nc-nd/4.0/.

\section{References}

1. Rahib L, Smith BD, Aizenberg R, et al. Projecting cancer incidence and deaths to 2030: the unexpected burden of thyroid, liver, and pancreas cancers in the United States. Cancer Res 2014;74:2913-21.

2. Siegel RL, Miller KD, Jemal A. Cancer statistics, 2020. CA Cancer J Clin 2020;70:7-30.

3. Hidalgo M. Pancreatic cancer. N Engl J Med 2010;362:1605-17.

4. Kamisawa T, Wood LD, Itoi T, et al. Pancreatic cancer. Lancet 2016;388:73-85.

5. Heinemann V, Haas $M$, Boeck S. Systemic treatment of advanced pancreatic cancer. Cancer Treat Rev 2012;38:843-53.

6. Kruger S, Ilmer M, Kobold S, et al. Advances in cancer immunotherapy 2019-latest trends. J Exp Clin Cancer Res 2019;38:268.

7. Brahmer JR, Tykodi SS, Chow LQ, et al. Safety and activity of anti-PD-L1 antibody in patients with advanced cancer. N Engl J Med 2012;366:2455-65.

8. Byrne KT, Vonderheide RH, Jaffee EM, et al. Special conference on tumor immunology and immunotherapy: a new chapter. AACR, 2015.

9. Banerjee K, Kumar S, Ross KA, et al. Emerging trends in the immunotherapy of pancreatic cancer. Cancer Lett 2018;417:35-46.

10. Foley K, Kim V, Jaffee E, et al. Current progress in immunotherapy for pancreatic cancer. Cancer Lett 2016;381:244-51.

11. Bilimoria KY, Stewart AK, Winchester DP, et al. The National Cancer Data Base: a powerful initiative to improve cancer care in the United States. Ann Surg Oncol 2008;15:683-90.

12. Boffa DJ, Rosen JE, Mallin K, et al. Using the National Cancer Database for outcomes research: a review. JAMA Oncol 2017;3:1722-8.

13. D'Agostino RB Jr. Propensity score methods for bias reduction in the comparison of a treatment to a nonrandomized control group. Stat Med 1998;17:2265-81.

14. Beatty GL, Chiorean EG, Fishman MP, et al. CD40 agonists alter tumor stroma and show efficacy against pancreatic carcinoma in mice and humans. Science 2011;331:1612-6.

15. Kimura Y, Tsukada J, Tomoda T, et al. Clinical and immunologic evaluation of dendritic cell-based 
immunotherapy in combination with gemcitabine and/ or S-1 in patients with advanced pancreatic carcinoma. Pancreas 2012;41:195-205.

16. Jackaman C, Majewski D, Fox SA, et al. Chemotherapy broadens the range of tumor antigens seen by cytotoxic CD8+ T cells in vivo. Cancer Immunol Immunother 2012;61:2343-56.

17. Liu WM, Fowler D, Smith P, et al. Pre-treatment with chemotherapy can enhance the antigenicity and immunogenicity of tumours by promoting adaptive immune responses. Br J Cancer 2010;102:115-23.

Cite this article as: Guddati AK, Komiya T, Patel SJ, Sharma N, Powell E. Impact of immunotherapy use in patients with stage IV pancreatic carcinoma. J Gastrointest Oncol 2020;11(4):654-662. doi: 10.21037/jgo-20-191
18. Tran TB, Maker VK, Maker AV. Impact of immunotherapy after resection of pancreatic cancer. J Am Coll Surg 2019;229:19-27. e1.

19. Kondo H, Hazama S, Kawaoka T, et al. Adoptive immunotherapy for pancreatic cancer using MUC1 peptide-pulsed dendritic cells and activated $\mathrm{T}$ lymphocytes. Anticancer Res 2008;28:379-87.

20. Lin M, Alnaggar M, Liang S, et al. An important discovery on combination of irreversible electroporation and allogeneic natural killer cell immunotherapy for unresectable pancreatic cancer. Oncotarget 2017;8:101795. 